\title{
Wireless sensor network for structural health monitoring: a contemporary review of technologies, challenges, and future direction
}

\begin{abstract}
The importance of wireless sensor networks in structural health monitoring is unceasingly growing, because of the increasing demand for both safety and security in the cities. The speedy growth of wireless technologies has considerably developed the progress of structural monitoring systems with the combination of wireless sensor network technology. Wireless sensor network-based structural health monitoring system introduces a novel technology with compelling advantages in comparison to traditional wired system, which has the benefits of reducing installation and maintenance costs of structural health monitoring systems. However, structural health monitoring has brought an additional complex challenges in network design to wireless sensor networks. This article presents a contemporary review of collective experience the researchers have gained from the application of wireless sensor networks for structural health monitoring. Technologies of wired and wireless sensor systems are investigated along with wireless sensor node architecture, functionality, communication technologies, and its popular operating systems. Then, comprehensive summaries for the state-of-the-art academic and commercial wireless platform technologies used in laboratory testbeds and field test deployments for structural health monitoring applications are reviewed and tabulated. Following that, classification taxonomy of the key challenges associated with wireless sensor networks for structural health monitoring to assist the researchers in understanding the obstacles and the suitability of implementing wireless technology for structural health monitoring applications are deeply discussed with available research efforts in order to overcome these challenges. Finally, open research issues in wireless sensor networks for structural health monitoring are explored.
\end{abstract}

Keyword: Wireless sensor network; Structural health monitoring; Power efficiency; High data rate and throughput; Fault tolerance; Time synchronization; Distributed processing; Scalability; energy harvesting; Mobile-SHM; Cloud-SHM 